\title{
Communication
}

\section{Search for new synthetic immunosuppressants II. Tetrazole analogues of hymenistatin $I^{\star \star}$}

Paweł Zubrzak ${ }^{1}$, Karol Kociołek ${ }^{1}$, Marek Smoluch ${ }^{2}$, Jerzy Silberring ${ }^{2}$, Marek L. Kowalski ${ }^{3}$, Barbara Szkudlińska ${ }^{3}$ and Janusz Zabrocki ${ }^{1}{ }^{凶}$

${ }^{1}$ Institute of Organic Chemistry, Technical University of Eódż, Eódż, Poland; ${ }^{2}$ Faculty of Chemistry, Jagiellonian University, Kraków, Poland; ${ }^{3}$ Department of Immunology, Medical University of Łódż, Łódź, Poland.

Received: 19 September, 2001; accepted: 7 December, 2001

Key words: hymenistatin I, cis-amide bond, 1,5-disubstituted tetrazole ring, immunosuppression

Linear and cyclic hymenistatin I (HS I) analogues with dipeptide segments $\mathrm{Ile}^{2}-\mathrm{Pro}^{3}$, $\mathrm{Pro}^{3}-\mathrm{Pro}^{4}$ and $\mathrm{Val}^{6}-\mathrm{Pro}^{7}$ replaced by their tetrazole analogues $\mathrm{Ile}^{2}-\Psi\left[\mathrm{CN}_{4}\right]-\mathrm{Ala}^{3}$, $\mathrm{Pro}^{3}-\Psi\left[\mathrm{CN}_{4}\right]-\mathrm{Ala}^{4}$ and $\mathrm{Val}^{6}-\Psi\left[\mathrm{CN}_{4}\right]-\mathrm{Ala}^{7}$ were synthesized by the solid phase peptide synthesis method and cyclized with the TBTU and/or HATU reagent. The peptides were examined for their immunosuppressive activity in the lymphocyte proliferation test (LPT).

A serious problem of transplantology, a dynamically expanding branch of contemporary medicine, is survival of the transplanted organ. A wider use of two existing and effective immunosuppressants, cyclosporin A ( CsA, sandimmun ${ }^{\circledR}$ ) [1] and FK-506 [2], as potent drugs for the prevention of graft rejection is limited by their side effects. In this situation, the search for new immunosuppressants exhibiting a similar mechanism of action but de-

\footnotetext{
${ }^{\star}$ Presented at the XVI Polish Peptide Symposium, September 1-4, 2001, Jagiellonian University, Kraków, Poland.

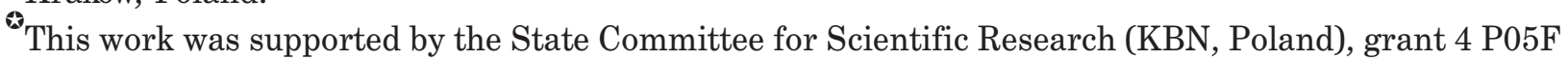
00413.

${ }^{\otimes}$ To whom correspondence should be addressed: Janusz Zabrocki, Institute of Organic Chemistry, Technical University of Łódź, 90-543 Łódź, Poland; tel. (48 42) 636 6588; fax: (48 42) 6365530 ; e-mail: zabrocki@ck-sg.p.lodz.pl
}

Abbreviations: ESI-MS, electrospray ionization mass spectroscopy; HATU, $O$-(7-azabenzotriazol-1-yl)- $N, N, N^{\prime}, N^{\prime}$-tetramethyluronium hexafluorophosphate; HS I, hymenistatin I; LPT, lymphocyte proliferation test; PHA, phytohemagglutinin; SPPS, solid phase peptide synthesis;TBTU, $O$-(benzotriazol-1-yl)- $N, N, N^{\prime}, N^{\prime}$-tetramethyluronium tetrafluoroborate. 
void of toxicity, especially in the group of naturally existing immunomodulatory peptides and their analogues, has become an important goal for medicinal chemistry.

Hymenistatin I [HS I] is a natural cyclic octapeptide of the sequence cyclo(Ile ${ }^{1}-\mathrm{Ile}^{2}-\mathrm{Pro}^{3}$ Pro $^{4}-$ Tyr $^{5}{ }^{5} \mathrm{Val}^{6}{ }^{6}$ Pro $^{7}{ }^{-} \mathrm{Leu}^{8}$-) isolated by Petit et al., in 1990 [3]. It has been shown by Siemion and coworkers [4] that hymenistatin I demonstrates some immunosuppressive activity in respect to both humoral (PFC) and cellular (DTH) immune response. A characteristic feature of this compound is the presence of a cis-amide bond between Pro $^{3}$-Pro ${ }^{4}$ residues, a $\beta$ VIa turn in the $\mathrm{Ile}^{2}-\mathrm{Tyr}^{5}$ region and a $\beta \mathrm{I}$ or $\beta \mathrm{II}$ turn in the $\mathrm{Val}^{6}$-Ile ${ }^{1}$ region in $\mathrm{CHCl}_{3}$ and $\mathrm{Me}_{2} \mathrm{SO}$ solution, respectively [3, 5].

To evaluate the significance of the $\mathrm{Il}^{2}-\mathrm{Pro}^{3}$, Pro $^{3}$-Pro ${ }^{4}$ and $\mathrm{Val}^{6}{ }^{-}$Pro $^{7}$ peptide bonds for HS I biological activity, we have synthesized three linear and three cyclic hymenistatine I analogues, with the dipeptide segments $\mathrm{Ile}^{2}-\mathrm{Pro}^{3}$, Pro $^{3}$-Pro $^{4}$ and $\mathrm{Val}^{6}{ }^{-}$Pro $^{7}$ replaced by their tetrazole analogues $\mathrm{Ile}^{2}-\Psi\left[\mathrm{CN}_{4}\right]-\mathrm{Ala}^{3}, \mathrm{Pro}^{3}$ $\Psi\left[\mathrm{CN}_{4}\right]-\mathrm{Ala}^{4}$ and $\mathrm{Val}^{6}-\Psi\left[\mathrm{CN}_{4}\right]-\mathrm{Ala}^{7}$, respectively.

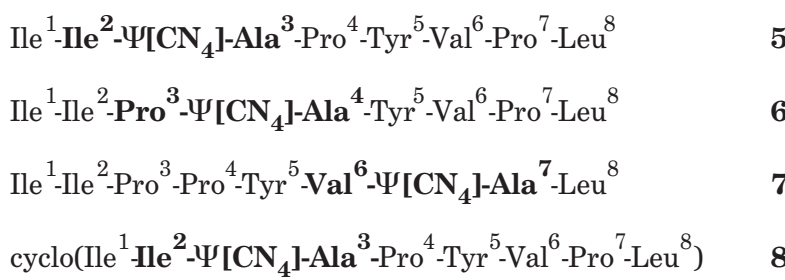

cyclo(Ile ${ }^{1}-\mathrm{Ile}^{2}-$ Pro $\left.^{3}-\Psi\left[\mathrm{CN}_{4}\right]-\mathrm{Ala}^{4}-\mathrm{Tyr}^{5}-\mathrm{Val}^{6}{ }^{6} \mathrm{Pro}^{7}-\mathrm{Leu}^{8}\right) \quad \mathbf{9}$

cyclo(Ile $\left.{ }^{1}-\mathrm{Ile}^{2}-\mathrm{Pro}^{3}-\mathrm{Pro}^{4}-\mathrm{Tyr}^{5}{ }^{5} \mathrm{Val}^{\mathbf{6}}-\Psi\left[\mathbf{C N}_{4}\right]-\mathrm{Ala}^{7}-\mathrm{Leu}^{8}\right) \mathbf{1 0}$

The 1,5-disubstituted tetrazole ring, $\Psi\left[\mathrm{CN}_{4}\right]$, has been proposed as a surrogate for cis-amide bonds, making it a valuable tool in the design of conformationally constrained peptidic receptor probes [6-10]. Since native hymenistatin I has been demonstrated to have only weak biological activity which precludes its use as an immunosuppressant we decided to look for immunosuppressive activity of hymenistatin I analogues, employing a standard test used for the assessment of immunosuppressive activity.

\section{MATERIALS AND METHODS}

The synthesis of desired peptides c(Ile $\left.-\Psi\left[\mathrm{CN}_{4}\right]-\mathrm{Ala}^{3}\right) \mathrm{HS}$ I 8, c(Pro ${ }^{3}-\Psi\left[\mathrm{CN}_{4}\right]-$ $\left.\mathrm{Ala}^{4}\right)$ HS I 9 and c(Val $\left.{ }^{6}-\Psi\left[\mathrm{CN}_{4}\right]-\mathrm{Ala}^{7}\right) \mathrm{HS}$ I $\mathbf{1 0}$ as well as the native compound was achieved by fragment condensation on a polymeric support (Merrifield type resin) using TBTU or HATU as a coupling reagent. The synthetic strategy is shown for peptide $\mathbf{8}$ as an example (Fig. 2). The tetrazole building blocks 4 was synthesized separately in solution (Fig. 1, Table 1) following a previously reported strategy $[8,9]$ and were used in the solid phase peptide synthesis procedure. The known proclivity of the C-terminal $\alpha$-carbon of tetrazole dipep-<smiles>[2H]C(NC(=O)OCC)C(=O)NC(C)C(=O)OCC</smiles>

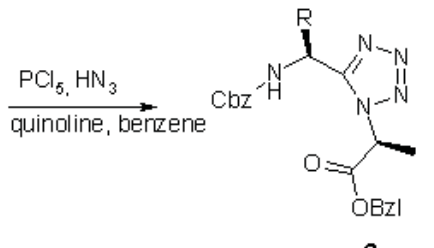

2

1. $\mathrm{HBr}, \mathrm{HOAC}$

2. MA, Boc-Xaa-OH $\mathrm{N}$-methylmopholine,<smiles>[R]C(NC(=O)[C@@H]([Tl])NC(=O)OC(C)(C)C)c1nnnn1[C@@H](C)C(=O)O</smiles>

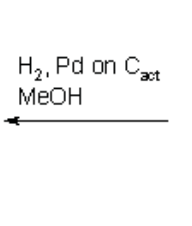

Figure 1. Scheme of tetrazole tripeptides synthesis. 
Table 1. Analytical data for tetrazole tripeptides

\begin{tabular}{|c|c|c|c|c|c|}
\hline Compound & Yield [\%] & $\begin{array}{c}{[\alpha]_{\mathrm{D}}(\mathrm{c}=1} \\
\mathrm{MeOH})\end{array}$ & $t_{\mathrm{R}}$ & $R_{\mathrm{f}}^{\mathrm{c}}$ & $M_{\mathrm{r}}^{\mathrm{d}}$ calc./found \\
\hline Boc-Ile-Ile- $\Psi\left[\mathrm{CN}_{4}\right]$-Ala-OBzl & 69.9 & -69.4 & $16.42^{\mathrm{a}}$ & $0.56^{1}$ & $514.6 / 515.1$ \\
\hline Boc-Ile-Pro- $\Psi\left[\mathrm{CN}_{4}\right]$-Ala-OBzl & 79.8 & -17.9 & $12.21^{\mathrm{a}}$ & $0.53^{1}$ & $498.6 / 498.9$ \\
\hline Boc-Tyr(2,6-diCl)-Val- $\Psi\left[\mathrm{CN}_{4}\right]-\mathrm{Ala}-\mathrm{Obzl}$ & 68.2 & -53.7 & $17.5^{\mathrm{a}}$ & $0.64^{1}$ & $697.6 / 698.2$ \\
\hline Boc-Ile-Ile- $\Psi\left[\mathrm{CN}_{4}\right]-\mathrm{Ala}-\mathrm{OH}$ & 95.2 & -54.8 & $5.46^{\mathrm{b}}$ & $0.64^{2}$ & $424.5 / 424.8$ \\
\hline Boc-Ile-Pro- $\Psi\left[\mathrm{CN}_{4}\right]-\mathrm{Ala}-\mathrm{OH}$ & 98.1 & -16.2 & $5.62^{\mathrm{b}}$ & $0.58^{2}$ & $408.4 / 409.1$ \\
\hline Boc-Tyr(2,6-diCl)-Val- $\Psi\left[\mathrm{CN}_{4}\right]-\mathrm{Ala}-\mathrm{OH}$ & 96.4 & -52.3 & $6.21^{\mathrm{b}}$ & $0.68^{2}$ & $607.4 / 607.9$ \\
\hline
\end{tabular}

${ }^{a}$ Analytical HPLC with linear gradient $50-70 \% \mathrm{~B} / 25 \mathrm{~min}$. (A) $0.05 \%$ trifluoroacetic acid in water, and (B) $0.038 \%$ trifluoroacetic acid in acetonitrile $/ \mathrm{H}_{2} \mathrm{O}(90: 10, \mathrm{v} / \mathrm{v}){ }^{\mathrm{b}}$ gradient $40-60 \% \mathrm{~B} / 25 \mathrm{~min} .{ }^{\mathrm{c}} \mathrm{TLC}$ systems: 1 = dichloromethane/acetone (30:1, v/v); 2 = butanol/acetic acid/water (4:1:1, by vol.). ${ }^{\mathrm{d}}$ Relative molecular mass calculated/found by FAB-MS measurements.

tides to epimerize [7-9] on prolonged base exposure prompted us to omit the neutralization step in the solid phase synthesis of peptides containing the tetrazole unit.

The cyclization of linear precursors was performed in solution using TBTU and/or HATU as a coupling reagent (Fig. 2). The structure of all peptides was confirmed by ESI-MS (Table 2).

All compounds demonstrated sufficient solubility and were tested for immunosuppressive activity by the lymphocyte proliferation test (LPT). Lymphocytes were isolated from pe-

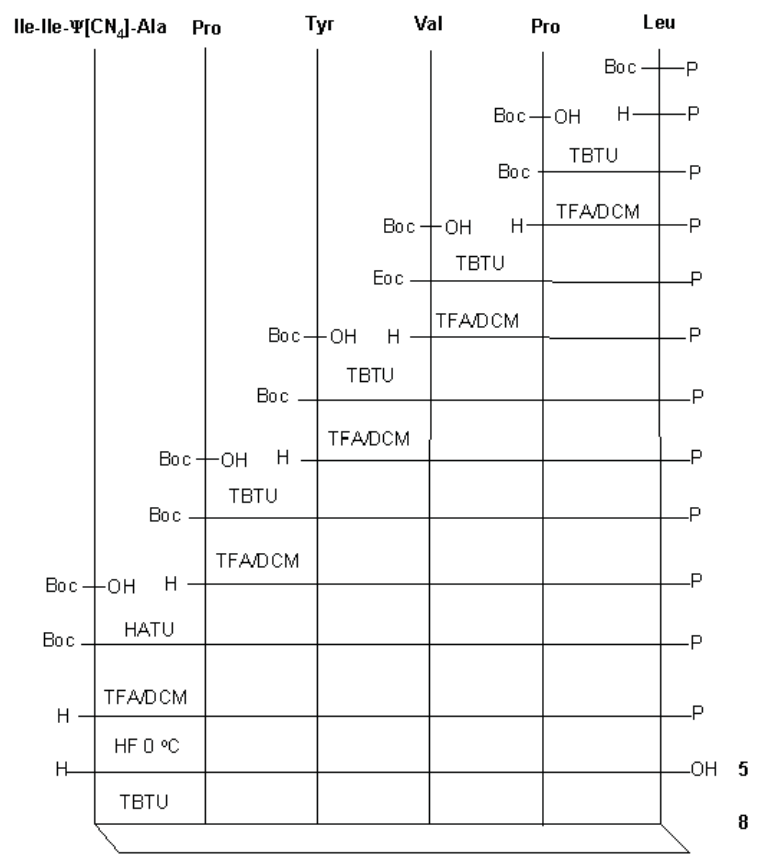

Figure 2. Scheme of c(Ile $\left.{ }^{2}-\Psi\left[\mathrm{CN}_{4}\right]-\mathrm{Ala}^{3}\right) \mathrm{HS}$ I synthesis. ripheral blood of healthy volunteers. Cells were stimulated with phytohemagglutinin (PHA) and incubated with different concentrations of the agents under study or cyclosporin A. Incorporation of $\left[{ }^{3} \mathrm{H}\right]$ thymidine, which was added to the cultures $24 \mathrm{~h}$ before cell harvesting, was determined in a liquid scintillation counter and expressed as counts per minute (c.p.m.).

\section{RESULTS AND DISCUSSION}

All synthesized analogues did not affect lymphocyte proliferation, thus are devoid of immunosuppressive activity as assayed by the LPT (Table 3). It was very surprising that even native hymenistatin I did not affect lymphocyte proliferation in this kind of biological test. On the other hand, our study does not exclude that the examined compounds could have an immunomodulatory effect on other immunological functions. In fact it has previously been noted that the native compound demonstrated some immunosuppressive activity in animal models (mice). However, the relevance of the biological activity of native hymenistatin I described in animal models by Cebrat et al. [4] to effective immunosuppression in humans is not clear and the lack of effect of the native compound on cytokine (IL-2 or IL-6) generation makes its clinical potential use unlikely. Unfortunately, as demonstrated 
Table 2. Analytical data for hymenistatin I analogues

\begin{tabular}{|c|c|c|c|c|}
\hline & Peptide & Yield $^{\mathrm{a}}(\%)$ & $t_{\mathrm{R}}^{\mathrm{b}}(\min )$ & $M_{\mathrm{r}}^{\mathrm{c}}$ calc./found \\
\hline 5 & Linear $\left(\mathrm{Ile}^{2}-\Psi\left[\mathrm{CN}_{4}\right]-\mathrm{Ala}^{3}\right) \mathrm{HS} \mathrm{I}$ & 83.1 & 6.37 & $910.07 / 909.87$ \\
\hline 6 & Linear $\left(\mathrm{Pro}^{3}-\Psi\left[\mathrm{CN}_{4}\right]-\mathrm{Ala}^{4}\right) \mathrm{HS} \mathrm{I}$ & 90.4 & 9.22 & $910.07 / 910.01$ \\
\hline 7 & Linear $\left(\mathrm{Val}^{6}-\Psi\left[\mathrm{CN}_{4}\right]-\mathrm{Ala}^{7}\right) \mathrm{HS} \mathrm{I}$ & 81.2 & 6.18 & $910.07 / 909.92$ \\
\hline 8 & $\mathrm{c}\left(\mathrm{Ile}^{2}-\Psi\left[\mathrm{CN}_{4}\right]-\mathrm{Ala}^{3}\right) \mathrm{HS} \mathrm{I}$ & 24.4 & 10.86 & $892.06 / 892.02$ \\
\hline 9 & $\mathrm{c}\left(\mathrm{Pro}^{3}-\Psi\left[\mathrm{CN}_{4}\right]-\mathrm{Ala}^{4}\right) \mathrm{HS} \mathrm{I}$ & 23.8 & 14.03 & $892.06 / 891.96$ \\
\hline & $\mathrm{c}\left(\mathrm{Val}^{6}-\Psi\left[\mathrm{CN}_{4}\right]-\mathrm{Ala}^{7}\right) \mathrm{HS} \mathrm{I}$ & 21.3 & 10.14 & $892.06 / 892.08$ \\
\hline
\end{tabular}

${ }^{a}$ Peptide content in crude product as shown by analytical HPLC with linear gradient $30-90 \%$ B/25 min. (A) $0.05 \%$ trifluoroacetic acid in water, and (B) $0.038 \%$ trifluoroacetic acid in acetonitrile $/ \mathrm{H}_{2} \mathrm{O}(90: 10, \mathrm{v} / \mathrm{v}){ }^{\mathrm{b}}{ }^{\text {gradient }}$ as above; ${ }^{\mathrm{c}}$ relative molecular mass calculated/found by ESI-MS measurements.

Table 3. Effect of the compounds studied on lymphocyte proliferation

\begin{tabular}{|c|c|c|c|c|c|}
\hline Compound studied & $\begin{array}{l}\text { Control solution } \\
\text { (PHA } 10 \mu \mathrm{g} / \mathrm{ml} \text { ) }\end{array}$ & $2.5 \mu \mathrm{g} / \mathrm{ml}$ & $5 \mu \mathrm{g} / \mathrm{ml}$ & $10 \mu \mathrm{g} / \mathrm{ml}$ & $20 \mu \mathrm{g} / \mathrm{ml}$ \\
\hline Linear $\left(\mathrm{Ile}^{2}-\Psi\left[\mathrm{CN}_{4}\right]-\mathrm{Ala}^{3}\right) \mathrm{HS} \mathrm{I}$ & 172505 & 178933 & 169858 & 168503 & 165870 \\
\hline Linear $\left(\operatorname{Pro}^{3}-\Psi\left[\mathrm{CN}_{4}\right]-\mathrm{Ala}^{4}\right) \mathrm{HS} \mathrm{I}$ & 172505 & 186371 & 188707 & 179547 & 187384 \\
\hline Linear $\left(\mathrm{Val}^{6}-\Psi\left[\mathrm{CN}_{4}\right]-\mathrm{Ala}^{7}\right) \mathrm{HS} \mathrm{I}$ & 172505 & 177748 & 166299 & 154650 & 163309 \\
\hline $\mathrm{c}\left(\mathrm{Ile}^{2}-\Psi\left[\mathrm{CN}_{4}\right]-\mathrm{Ala}^{3}\right) \mathrm{HS} \mathrm{I}$ & 200864 & 207386 & 199482 & 215386 & 227716 \\
\hline $\mathrm{c}\left(\mathrm{Pro}^{3}-\Psi\left[\mathrm{CN}_{4}\right]-\mathrm{Ala}^{4}\right) \mathrm{HS} \mathrm{I}$ & 200864 & 206011 & 213888 & 207746 & 215366 \\
\hline $\mathrm{c}\left(\mathrm{Val}^{6}-\Psi\left[\mathrm{CN}_{4}\right]-\mathrm{Ala}^{7}\right) \mathrm{HS} \mathrm{I}$ & 200864 & 207154 & 202170 & 212090 & 220303 \\
\hline Native HS I & 200864 & 204662 & 211458 & 217956 & 224532 \\
\hline
\end{tabular}

Results are shown in c.p.m. (counts per minute)

in our study, modification of hymenistatin I by a 1,5-disubstituted tetrazole ring did not improve its immunosuppressive activity in vitro to any significant degree.

\section{R E F E R E N C E S}

1. Ellis, G. \& West, G.B. (1988) Progr. Med. Chem. 25, 1-33.

2. Sigal, N.H. \& Dumont, F.J. (1992) Annu. Rev. Immunol. 10, 519-560.

3. Petit, G.R., Clewlow, P.J., Defresne, C., Doubeck, D.L., Cerny, R.L. \& Rutzler, K. (1990) Can. J. Chem. 68, 708-711.

4. Cebrat, M., Wieczorek, Z. \& Siemion, I.Z. (1996) Peptides 17, 191-196.

5. Konat, R.K., Mierke, D.F., Kessler, H., Kutscher, B., Bernd, M. \& Voegeli, R. (1993) Helv. Chim. Acta 76, 1649-1666.
6. Marshall, G.R., Humblet, C., Van Opdenbosch, N. \& Zabrocki, J. (1981) in: Peptides: Synthesis, Structure and Function, Proc. 7th Am. Pept. Symp. (Rich, D. \& Gross, E., eds.) pp. 669-672, Pierce Chemical Co., Rockford IL.

7. Yu, K.-L. \& Johnson, R.L. (1987) J. Org. Chem. 52, 2051-2059.

8. Zabrocki, J., Smith, G. D., Dunbar, J.B., Jr., Iijima, H. \& Marshall, G.R. (1988) J. Am. Chem. Soc. 110, 5875-5880.

9. Zabrocki, J., Dunbar, J.B., Jr., Marshall, K.W., Toth, M.V. \& Marshall, G.R. (1992) J. Org. Chem. 57, 202-209.

10. Smith, G.D., Zabrocki, J., Flak, T.A. \& Marshall, G.R. (1991) Int. J. Pept. Prot. Res. 37, 191-197. 